\title{
CONCEPTS AND ACHIEVEMENTS EXPERIMENTAL MODELS (CYBER-MIXMECHATRONICS SYSTEMS) FOR SMART COBOTIC PLATFORMS IN THE SMART INDUSTRY (4.0)
}

\author{
Gheorghe Gheorghe \\ ${ }^{1}$ National Institute of Research and Development for Mechatronics and Measurement \\ Technique - INCDMTM, Bucharest, Romania \\ Email: geocefin@yahoo.com
}

\begin{abstract}
The scientific work presents some constructive solutions of mechatronic multiapplication robotic platform, СOBOT type used for ultra-precisely remote monitoring and remote control for digital enterprise and Smart Industry (4.0). The paper also positions the IT\&C technology for the digital enterprise and Smart Industry (4.0), with some examples of experimental models for intelligent platforms СОВОТ type.
\end{abstract}

Keywords: Cyber-Mixmecatronics Systems; Cobotic Intelligent Platforms; Intelligent Industry.

\section{Introduction}

\subsection{Future Ideas for Intelligent Cobotic Platforms \\ Cobotics systems for Electronics Manufacturing for integrated tactile adaptive control}

Industry 4.0, the Internet of Things, cloud computing, self-management, e-health, augmented reality or artificial intelligence benefit from advanced technology, in the sense that the semiconductor and electronics industries make them available today.

At the same time, these growing markets present great challenges: even smaller components, with a higher functional density, shorter innovation cycles and an increasing diversity of smart end products.

The precision, efficiency, reliability and flexibility of production systems are increasingly decisive at the level of board and chip, not to mention at the level of printed circuit boards.

Each positioning solution for the placement, alignment, processing or inspection of components must be able to meet these requirements.

Process type, duty cycle, tool types, machining area size, feature size and criteria such as flow rate, accuracy and geometric tolerances produce different requirements on technological motion systems.

Many years of experience in the semiconductor market, technological diversity and the high range of vertical production allow it to react flexibly to market development and provide motion systems that meet the needs, integrators and end users in the field of electronics production.
This scientific paper contains examples of highperformance cobotic systems for different stages of production, from standard products to highly customized solutions that communicate through standard industrial networks.

Figure 1 shows an example of an integrated tactile adaptive control application of a mouse button and the toutch-screen surface of a tablet in order to test both functionality and reliability (average operating time - MTBF).

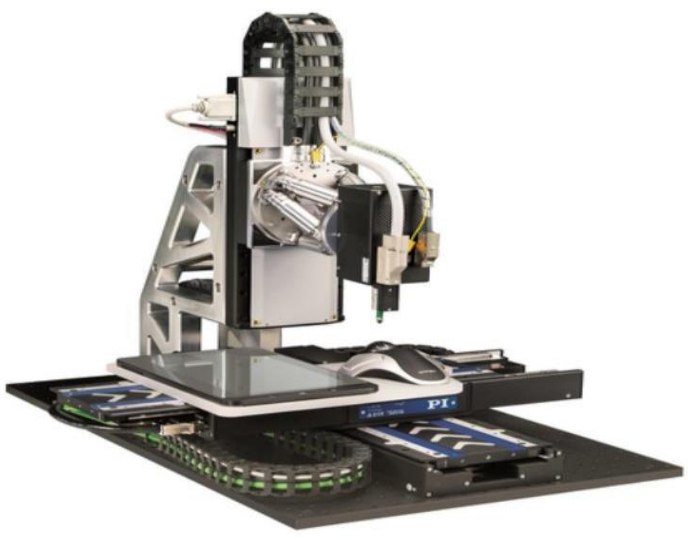

Figure 1

It provides technologically complex solutions and therefore uses its own high-precision drive and positioning components.

All levels of integration can be offered up to multi-axis combinations that use different drive technologies, including motion control.

The engineering projects of the motion subsystems were part of the basic activities as customized solutions, perfectly fitting into existing 
processes, advanced automation in manufacturing and inspection processes for the production of chips or optical-photonic cables, as well as complex research facilities.

The cobotic axle system has high displacement accuracy and load capacity due to precision linear guides with preloaded recirculated ball bearings provided with $2 \mathrm{~mm}$ pitch compensated ball screw.

The base is aluminum for maximum stability.

Types of actuation and position measurement for the following models are:

- DC motor with integrated high-speed ActiveDrive amplifiers combined with directencoding linear encoder for high resolution and increased repeatability.

- also with integrated support brake.

- direct gear motor with direct control ActiveDrive integrated and rotary encoder.

- DC motor with integrated amplifier and rotary encoder.

- Brushless DC motor with integrated block switch and rotary encoder.

As specifications we have:

- displacement ranges 102, 204 and 306 mm (4 ", $8 ", 12$ ")

- speed up to $100 \mathrm{~mm} / \mathrm{s}$

- bidirectional repeatability $\pm 0.2 \mu \mathrm{m} \mathrm{n}$ Load capacity per $100 \mathrm{~kg}$

- ball screw with zero play

- possible XY and XYZ configurations
The areas of application are: Industry and Research. Laser material processing, surface inspection, etc.

Tactical and optical testing of electronic devices, regarding the control of machines with assembled electronic components

Electronic devices are getting smaller, more complex, working in different environments and include several types of components that require tactile, optical, and electronic testing methods. The position of these devices in the final assembly requires the precise positioning of the inspection instrument in several degrees of freedom. Powerful flexible motion systems are offered for different test methods that can be integrated into customers' production lines while maintaining capacity and efficiency.

The key features of cobotic motion systems are:

- the unique range of motion technologies to design the most advanced solutions;

- high-performance industrial motion control platform ensures coordinated movement of all axes for maximum efficiency

- easy connection and management of sensors and test devices with flexible connectivity;

It can be integrated as a subsystem in most PLC's and automated controllers.

According to Figure 2, the following scenario is described:
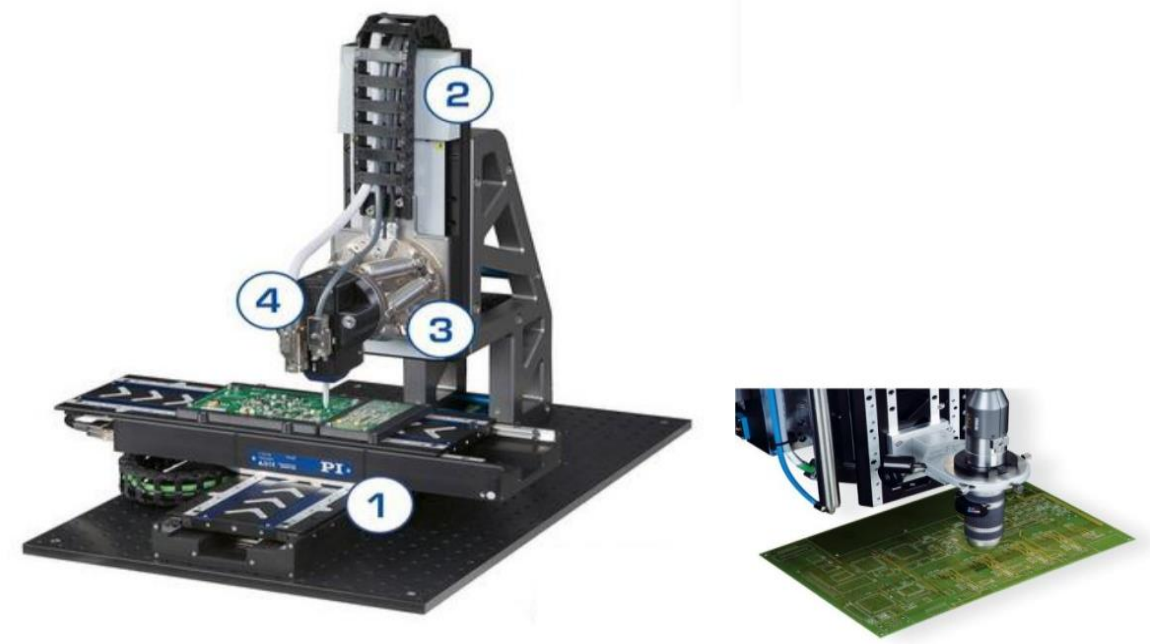

Figure 2

(1) Positioning the part

- Linear motors with direct magnetic drive for high precision and speed

- Absolute encoder with $1 \mathrm{~nm}$ resolution

- XY cable chain management

- Precision linear motor stage v451
(2) Positioning of test devices

- Linear motor with ring screw or voice coil

- Cable management integration

- Brake or counterbalance

- Direct linear encoder measurement

- (M511) High precision linear stage 
(3) Positioning the sensor at different angles

- Parallel kinematic hexapod

- Six degrees of freedom

- Freely definable center of rotation

- (H 811-6) miniature hexapod ax

(4) Force control measuring sensor

- PiMag Voice call linear actuator

- Force sensor with $1 \mathrm{Mn}$ resolution

- Weight force compensation

- Voice call linear actuator

Electronic component manufacturers require high control wiring efficiency with assembled electronic components. They can take advantage of the high speed of automation systems based on the integration of hexapodal robots, but also of the high precision offered for them in the active control of the production processes in the electronics industry.

Accuracy and reliability in a large number of operating cycles are also important.

Cobotic motion platforms meet these requirements and also ensure easy connectivity to other automation systems and devices in the production line.

Thanks to the precision linear guides with crossed bearings, the linear positioning is especially suitable for scanning applications with constant speed.

The anti-creep system reliably prevents loading into the joints.

The guides have a high load capacity and precision in all operating conditions.

The position measurement is performed with the highest accuracy directly on the movement platform, so that the nonlinearity, the mechanical play or the elastic deformation do not influence the measurement.

The high-resolution PIOne encoder was developed with proper processing of the measured values and allows a position resolution of much less than one nanometer.

Optical and non-contact PIOne encoders are based on an interferometric measurement principle. Due to the short signal period and high signal quality, the linearity error of PIOne encoders is less than 1\%. PIOne encoders support direction detection when they detect a reference signal.

Absolute encoders provide explicit position information that allows immediate position determination. This means that the reference is not required during start-up, which increases efficiency and safety during operation.
Direct drive motors are suitable for positioning tasks with the highest accuracy requirements, as there is no unwanted interaction with permanent magnets.

This allows smooth running even at the lowest speeds and at the same time there is no vibration at high speeds.

Non-rectilinearity in control behavior is avoided and any position can be easily controlled. The actuating force can be easily set.

In direct drives, the force of the drive element is transmitted directly to the load to be moved without the use of mechanical transmission elements, such as the coupling, the drive screw or the reducer.

The multi-axis system can be controlled by an ACS motion control module for optimal axis synchronization. ACS Motion Control offers a complete modular system and organizes components into three different layers:

- the first level is the user interface. this is basically the host software and communication with the motion system is allowed.

- second level devices are called motion controllers. The motion controller is responsible for communicating with the host software and also takes care of everything related to profile generation, trajectory, macros, diagnostics, etc.

- position commands are sent to the universal drive modules at the third level via an industrial network. The motion controller, units and power supplies are integrated in a single housing. These products are called control modules.

The drive modules power and drive the motors, manage the feedback devices, manage the digital inputs and outputs ( $\mathrm{I} / \mathrm{O}$ ) and analyze the sensor signals for closed loop positioning

As applications Industry and Research applications. Automation. Metrology. Photonic and precision scanning in the manufacture of electronic circuits.

\section{Cobotic system with integrated hybrid hexapod}

The electronics industry often requires very specific solutions, for example, for flexible band connections. Cobotic systems, (fig. 3) represent an ideal basis for customized adaptations. 


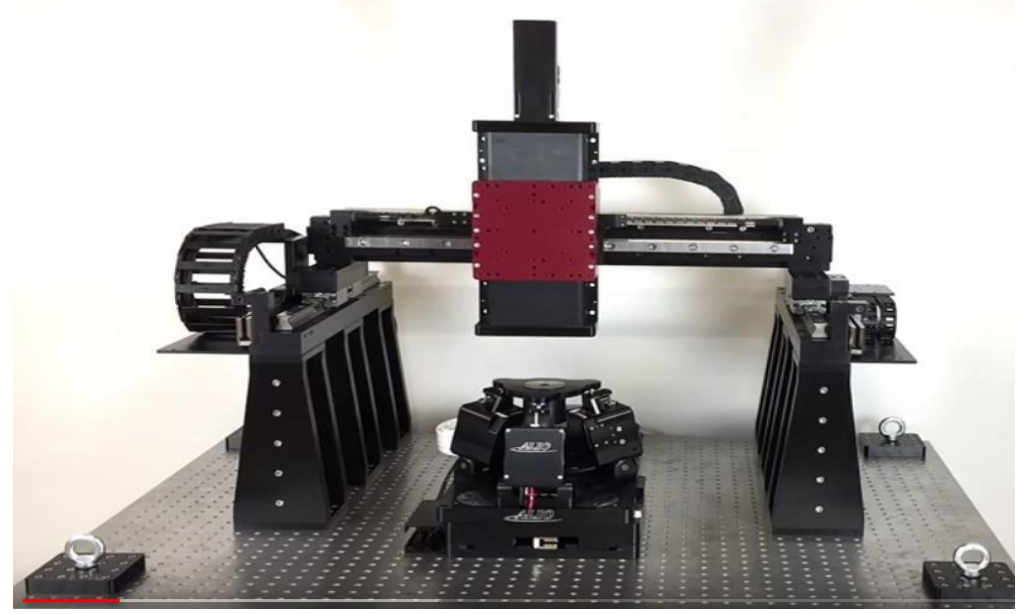

Figure 3

The stacking width and height can be varied almost arbitrarily, the configuration of the motors ensures extremely high accelerations of up to $5 \mathrm{~g}$, thus ensuring the highest productivity depending on the application.

The features of ACS motion controllers, such as "Input Shaping", help build a stable and adaptive system that actively suppresses possible vibrations.

Positioning accuracy requirements are particularly high in the production and inspection of semiconductors or circuit boards. The high dynamics of the axis of motion is necessary for an optimized flow of the processed wiring. In this case, a travel range of $200 \mathrm{~mm} \times 200 \mathrm{~mm}$ in $\mathrm{XY}$ is usually sufficient. All guides have special bearings that guarantee the best flatness for a precise and high dynamic positioning of the movement. In addition, it is possible to maintain a stable position

The series of applications is offered in the range of displacements on axes of $500 \mathrm{~mm}, 1000 \mathrm{~mm}$ and $1500 \mathrm{~mm}$. Custom lengths are available by direct order at the factory. With speeds of up to $1 \mathrm{~m} / \mathrm{sec}$, cobotic systems are useful for applications that require high-speed positioning of light payloads.
The series of cobotic systems is standard with stepper motors. The motors are also available with 4000 rotations, with incremental optical rotary encoders, mounted integral with the motor.

The mechanical limit switches of the travel end are standard on the axle travel system.

The drive system uses a $9.5 \mathrm{~mm}$ wide guide. The mobile system moves on four pre-loaded ball bearings, profiled to fit the contour of the aluminum extrusion base to provide a larger contact surface.

All cobotic systems are machined from AL 6061 aluminum alloy and black anodized. The base is an aluminum extrusion with a clear anodized finish.

Typical applications for a cobotic system include pick \& place, component inspection and distribution.

\section{- Hermetic sealing of electronic omponents using laser welding}

Fig. 4 shows a solution for hermetically sealing electronic components with laser welding.



Figure 4 
On a cobotic platform Hexapod type 6-Axis Miniature, is fixed a motor with torque control and an LCM system - Laser Control Module

Torque-controlled motors are directelectromagnetic motors.

It is distributed with mechanical traction components and transfers the driving force directly and without friction to the movement platform.

The specifications of this cobotic system are:

- active axis $\theta \mathrm{Z}$

- travel range $>360(1)^{\circ}$;

- integrated Sin / cos sensor;

- Peak Sensor Resolution 1845 Lines;

- revolution Minimum incremental movement 2 $\mu$ rad Type;

- unidirectional repeatability 4 rad Type;

- bidirectional repeatability $88 \mu \mathrm{rad}$ Type;

- angular speed 500 rpm Max;

- Optical reference switch, coding index, $5 \mathrm{~V}$, TTL differential, $1 \times$ on the platform revolution.

The mechanical properties of the cobotic system are:

- cross roller bearings;

- load capacity / axial force $20 \mathrm{~N}$ Max. ;

- permissible lateral force $6 \mathrm{~N}$ max .;

- moment of inertia $0.00025 \mathrm{kgm}^{2} \pm 20 \%$;

- nominal moment 0.49 (2) 0.49 (2) N • mMax .;

- rated current 2.05 (2) 2.05 (2) A Typ .;

- maximum moment 1.25 (2) 1.25 (2) N • mMax .;

- maximum current (max. 6 s) 5 (2) 5 (2) A Max .;

- torque constant $0.240 .24 \mathrm{~N} \bullet \mathrm{m} / \mathrm{A}$ Type;

- resistance phase phase $33 \Omega$ Type. Inductance phase $0.50 .5 \mathrm{mH}$ Type;

- number of pairs of poles 12 12;

- electromagnetic safety brake.

The LCM system is an EtherCAT network module that allows ACS controllers and control modules to control a laser source directly with full motion synchronization, resulting in the highest quality laser micro-processing and optimum efficiency.

It is compatible with interfaces from a wide range of lasers for complex applications such as DPSS, CO2, excimer, fiber, Q-switch, picosecond, femtosecond and more.

The system has several operating modules:

- Power control module:

- fixed frequency;

- fixed pulse width;

- fixed service cycle;

- Tickle mode;

- fixed frequency and pulse width.

- Motion capabilities (with ACS Controller):

- fast point positioning on a single axis or on several axes using 3rd order interpolation;

- up to 5 axes, movement traversed by means of the universal G code or the native ACS XSEG motion commands, with advanced forward and rounding algorithms;
- mapping compensation error;

- support for Cartesian and non-Cartesian systems.

The functional summary covers:

- digital pulse modulation for dynamic control of laser power;

- synchronization of the pulse with the XYZ movement path in certain positions;

- synchronization by starting / stopping the laser gate with the XYZ movement path in specific positions;

- flexible electrical interface: active laser output, safety lock input, error input for robust safety implementation, general purpose programmable I / 0.

When faced with health problems, today's medical technology makes it possible for implants, such as pacemakers or insulin pumps, to perform important functions. Such implants are extremely complex systems consisting of electrical and electronic components. Hermetic sealing, which keeps the microsystem and surrounding tissue reliably separated from each other, is necessary for safe and reliable use in the human body. Components depend on airtight seals for them to work properly and safely in other industries, for example, sensors and electronics in the semiconductor industry or the charging of batteries must be protected against vapors or foreign particles.

Laser welding is a method used for sealing. For this purpose, the laser point is focused on the rotating workpiece. In order to obtain a uniform welding quality, the size of the laser point must be maintained keeping the same distance between the laser and the workpiece in the same way during welding. In addition, the laser beam must strike the surface of the part at the correct angle. Part retention or alignment errors must also be considered and corrected by controlling the laser welding path.

Hexapods, which can move in six degrees of freedom and allow the definition of reference systems for the workpiece and instrument ("work", "instrument" coordinate systems), can reliably perform all these tasks.

\section{Simulation and Verification of the Stability of the Image Taken by a Drone}

Taking high-definition images from drone systems, despite poor lighting conditions, taking snapshots without fading, recognizing traffic signs or road markings in driver assistance systems, or identifying dangerous situations in surveillance systems - all of these are possible today with the help of modern cameras. But how good is the still and video image quality of a camera or smartphone? 
End-users, manufacturers from companies in the aerospace, medical and security sectors or in the field of automation technologies - all require high performance capabilities and are looking for answers to these questions. Companies that deal with these issues rely on test equipment that guarantees reliable and comparable test results. For testing image stabilization systems of cameras that process images from drones, for example, the frequencies and movements of the axes must be simulated realistically and must be identical for each test.

In order to perfectly adapt the trajectory to the requirements of the application, it is possible to define different coordinate systems using a Hexapod cobotic platform as in figure 5 .

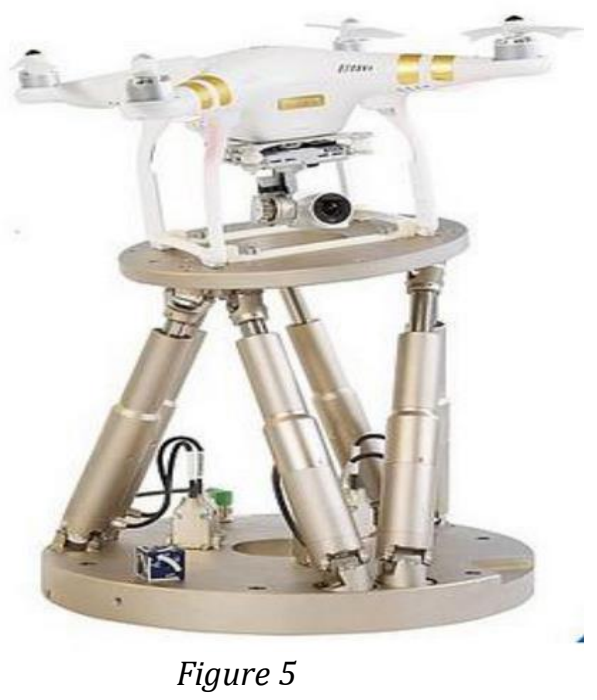

This offers great advantages for applications in industrial automation, but also for drone flight simulation.

The frequency of the in-flight tremor, or of the human hand during filming, is around $12 \mathrm{~Hz}$, which means that the tests must be able to cover a range between 0 and $12 \mathrm{~Hz}$. Most of the required frequencies are between 4 and $8 \mathrm{~Hz}$. Hexapod systems can be used successfully to achieve this goal.

An effective testing procedure is of particular interest to both manufacturers and users. And here is a key role of Image Engineering. The main experience is the measurement of digital image quality, as well as the development of appropriate testing procedures and complete testing systems for cameras.

Image quality assessment and image stabilization testing place high technical requirements on the test procedure and its components. Above all, the goal is to accurately simulate the tremor by realistic simulation of the drone's flight.

Hexapod platforms are used for precise movement and positioning, alignment and displacement of loads in all six degrees of freedom, ie three lines and three rotating axes. Hexapods have a parallel kinematic structure, meaning that the six units act together on a single moving platform.

The length of the motion actuators is variable, allowing the system to move in all six degrees of freedom in space. This special design of the hexapod optimizes the overall rigidity of the system and allows more space for movement.

Depending on their design, hexapods can position loads from a few $\mathrm{kg}$ to a few hundred $\mathrm{kg}$ or even a few tons in any spatial orientation, ie independent of the mounting orientation and with high accuracy.

Motion simulation applications benefit of:

- more degrees of freedom than the classic solutions;

- pivot point that can be defined depending on the position of the image stabilization component in the camera;

- following the predetermined trajectories;

- movement frequencies at $50 \mathrm{~Hz}$;

- CIPA certification.

For the CIPA certification:

The lobby association of the Japanese photographic industry Camera \& Imaging Products Association (CIPA) sets standards for motion systems that are used in image stabilization test equipment.

Hexapods are the only six-axis positioning system for simulating camera vibration during in-flight shooting that meets the requirements of the CIPA standard.

The freely definable rotation or pivot point is an essential feature of the parallel-kinematic hexapod. This means that the movement of the hexapod platform can be specifically adapted to the position of the gyroscope mounted on the camera, so that ideal realistic conditions can be created by flight simulation and real-time image acquisition.

Hexapods can be designed considerably more compactly than multi-axis positioning systems in series. Because only one platform is operated, most often with a large aperture, the moving mass of the Hexapod is significantly smaller. This leads to improved dynamics, with a considerably faster response. In addition, the electric cable with the control signals from the controller does not create any problems, so no additional forces and torques are present so as to reduce the accuracy.

In the case of stacked systems (dodecapod systems), the lower axles not only move the mass of the payload, but also the mass of all other units that follow. This reduces the dynamic properties and total rigidity of the system.

The "mini Hexapod" Hexapod system is used for testing smartphones and the "universal Hexapod" system is used for heavier cameras. Both are perfectly suitable for testing image stabilization systems and are already CIPA certified. "CIPA certification allows us to take a pioneering role in 
qualifying a six-axis system for evaluating image stabilization systems," says Wüller.

Meanwhile, both Hexapod systems have proven to be practically practical as follows:

- mini Hexapod can simulate vibrations, e.g. rotary motion, with dynamics of $20 \mathrm{~Hz}$ and a deviation of $0,1^{\circ}$. For this purpose, the parallelkinematic system performs a repeatable and defined test trajectory. It offers travel distances of up to 34 $\mathrm{mm}$ in the $\mathrm{XY}$ plane and up to $13 \mathrm{~mm}$ in the $\mathrm{Z}$ direction. Tilt angles are $20^{\circ}$ around the $\mathrm{X}$ and $\mathrm{Y}$ axes and up to $42^{\circ}$ around the vertical.

- the universal Hexapod system has higher loads, provides travel distances of up to $100 \mathrm{~mm}$ and rotation angles of up to $60^{\circ}$.

\subsection{Cobotic Robotic Station}

Today's industry managers are under significant pressure to automate production processes to make products that can evolve rapidly as the needs of the customers they want to serve. Consequently, we see that car platforms, for example, with a shelf life of three years instead of five, are becoming the norm, and it is observed that electronic products adopt an obsolescence program of one to two years instead of three years. This new reality significantly disrupts the way final products and supporting equipment (eg machines) are designed and manufactured.

At the highest level, a shorter product life cycle means that the depreciation window for related production equipment is also shorter, creating a need for production assets that can be designed, deployed and reused at low cost. Despite this pressure, custom industrial equipment is still designed and manufactured using traditional methods, based on an extremely fragmented industrial ecosystem of regional industrial distributors, engineering integrators and local machine shops. This industry is ripe for a major change through the use of collaborative robots (Fig. $6)$.



For the description are produced (fig. 6) systems based on the use of a Universal Robot - UR3 framed in a workspace with $450 \times 540 \mathrm{~mm}$ aluminum motherboard, (as in Figure 6). The height of the table is $720 \mathrm{~mm}$, with an adjustable leveling support of 80 $\mathrm{mm}$. There is a high shelf for the cabinet with electronic control circuits and modules.

The technical specification is: - weight: $35.2 \mathrm{~kg}$

\section{Cobotic Application - Collaborative Robot}

The collaborative robot - OMRON TM in Figure 7 is designed to work with both humans and machines, this robot is the latest in a long line of innovations in automation technology for industrial applications.

Safe and transportable, with integrated vision, this robot allows quick start and quick adaptation.

Its intuitive software allows the robot to be easily trained to perform almost any task, wherever it is needed, freeing the workforce from repetitive tasks and increasing productivity.

Regardless of the industrial field, in automobiles, semiconductors, secondary packaging for food or cosmetics, the OMRON TM collaborative robot is designed to adapt to almost any industry.

The OMRON TM collaborative robot offers a vision system with a built-in camera. The integrated camera locates objects in a wide field of view, and image enhancement light allows objects to be recognized in almost any condition.

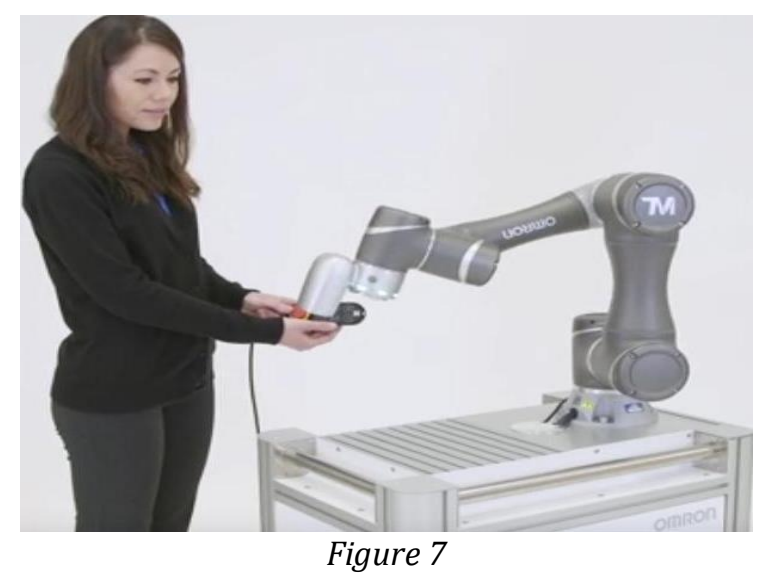

The vision system improves reliability, consistency and high-precision placement and includes features such as pattern matching, barcode reading and color classification that allow inspection, measurement and sorting at no extra cost or effort.

With the various vision features and simple and intuitive programming environment of OMRON TM collaborative robots, it allows for continued safe, flexible and collaborative manufacturing between man and machine.

As changing consumer trends continue to reduce product life cycles, manufacturers need production 
lines to meet the requirements of frequent product changes. In addition, as labor shortages become a reality, manufacturers are striving to automate simpler and more monotonous tasks, to create jobs where people can contribute to more creative tasks.

The RobotTM series offers a unique solution to easily install a robot to automate applications such as assembly, lifting, placement and inspection. At the launch of the TM series, there is a "mobile compatible" model, which will integrate perfectly into the market of an autonomous mobile robot. This allows users to automate more complex tasks, such as retrieval and placement on a tray or container, and connect processes with autonomous mobile robots.

OMRON Robots from TM series have the following specifications:

- arm length: 700 mm, 900 mm, 1100 mm, 1300 $\mathrm{mm}$;

-payload: 4 kg, $6 \mathrm{~kg}, 12 \mathrm{~kg}, 14 \mathrm{~kg}$;

- power supply: AC, DC;

- according to SEMI S2 Safety Guides.

Three key features of the TM series collaboration robot are:

(1) Intuitive programming interface reduces programming time: Reduced installation and configuration times compared to traditional industrial robots. The intuitive programming interface based on the flowchart and easy teaching requires little or no previous robot programming experience.

(2) The integrated arm view system reduces setup time: the TM series is equipped with built-in vision and integrated lighting, allowing the user to capture products with a wide viewing angle. Equipped with image detection features such as pattern matching, barcode reading and color identification, this robotic system makes inspection, measurement and sorting applications extremely easy to configure from the control panel.

(3) according to all safety standards of collaborative robots, reducing installation time: the TM series meets all safety standards that allow cooperation between humans and machines and can be operated safely around humans without the necessary classic industrial safety "fences" traditionally for industrial robots, again greatly reducing installation time. (According to the safety requirements for ISO10218-1 industrial robots, as well as the safety requirements for ISO / TS15066 collaborating industrial robots)

With the addition of the TM series collaborative robot to the manufacturing line, safe, flexible manufacturing and human-machine collaboration are allowed to accelerate the implementation of the
"Flexible Innovation-Automation" concept, an innovative manufacturing concept.

\subsection{Cobot - Transcranial magnetic stimulation}

An increasing number of patients increasingly need to free the specialist operator (doctor or nurse) from a time-consuming and time-consuming task and to reallocate some of his time to value-added tasks. bigger.

Finally, avoiding any movement of the head during a period of 30 s, even at rest and relaxation, is a real constraint for the patient.

The objective of the "TMS-Cobot" system shown in Figure 8 is to provide an affordable robotic solution, with head movement compensation, to improve the accuracy of a medical procedure, while providing the operator with avoidance of a demanding and repetitive task. and reducing unpleasant constraints for the patient (through mechanical stiffeners or other methods).

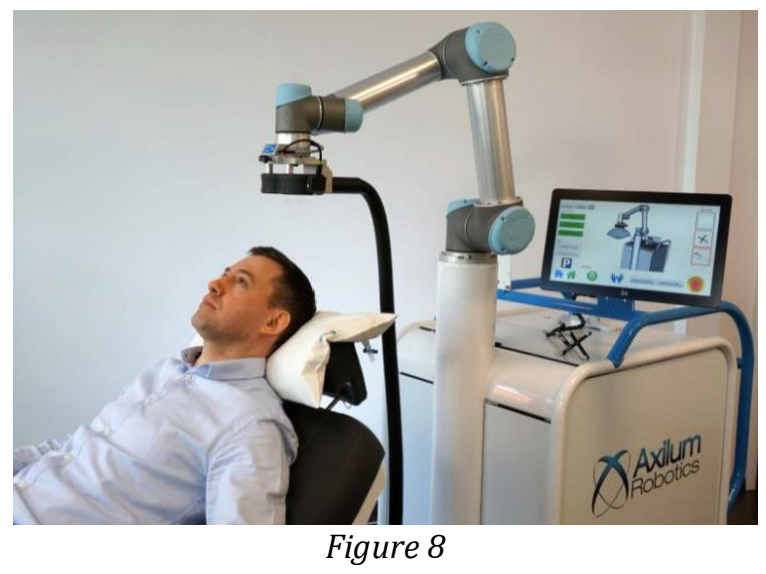

For example, it is important for the physician to maximize the ability to provide the stimulation dose with electromagnetic radiation provided by a coil and an electric current at the appropriate and predetermined location and with TMS-Cobot is possible.

The key features are:

- collaborative robotic "cobot" technique (safety);

- such technology offers intrinsic safety features through a collision detection mechanism at each common level, allowing close collaboration with the patient and the doctor;

- the movements of the robotic arm can be automated or directed manually by the user who has activated a so-called initial free action mode;

- maintaining the position and orientation of the Transcranial Magnetic Stimulation (TMS) coil during the therapy session by optically tracking the markers on the head;

- compensates for the potential movement of the head during the therapy session; 
- ensures a permanent contact between the coil and the head (integrated pressure sensor);

- compatible with any seat;

- Easy and extremely mobile: Can be easily moved on its wheels.

Ergonomics: when piloted by a compatible neuronavigation system, the operator controls the TMS-Cobot from the graphical user interface of the neuronavigator

When piloted by the Axilum Robotics Optical Tracking System, the operator controls the TMSCobot from a control panel.

The key advantages are:

- improves the accuracy and repeatability of the procedure;

- the accuracy of the robotic arm is less than 2 $\mathrm{mm}$;

- offers the operator relief from a repetitive and time-consuming task;

- Once the therapy session has been initiated, minimal operator attention is required to ensure proper position, orientation and contact of the coil on the patient's head.

Cobot cmpatibility:

-TMS-Cobot is compatible with therapy coils from Deymed, Magstim, MagVenture, Mag \& More, Neurosoft. List of coils compatible on request

- TMS-Cobot can be piloted either by Axilum Robotics Optical Tracking System (without MRI) or by a compatible neuro-navigation system from Localite, Rogue Research / Brainsight, Syneika

The dimensions and weight for cobot:

-TMS-Cobot fits any space and fits most elevators due to its practical size and light weight;

- height with arm: (H) 1770 mm;

- basic dimensions: (L x W) 1111 x 650 mm;

- weight: $143 \mathrm{~kg}$;

- regulatory information;

- CE (EU) marking.

TMS-Cobot is a Class IIa medical device designed to automate and improve the accuracy and repeatability of the positioning of a Transchannel Magnetic Stimulation Coil (TMS) in clinical situations where the use of compatible devices is desired, except for peripheral nerve stimulation. It bears the CE marking 0120, the conformity assessment of which has been established by the notified body SGS. These medical devices are not reimbursed by the French health insurance.

Axilum Robotics TMS-Cobot $\AA$ TS MV is indicated for the positioning and spatial orientation of the treatment coil of the MagVenture TMS Therapy system.

Transcranial magnetic stimulation is used to treat major depressive disorder in patients who have not responded to medication. Proper positioning of the coil so that it accurately targets the correct part of the brain is the key to effective treatment.
Patient movement can interfere with correct positioning, but the TMS-Cobot TS MV is an optical system capable of tracking the patient's movement and continuously adjusting its positioning to keep the coil focused on the target. The arm can be adjusted and repositioned by the operator as needed.

\subsection{Example of male / female positioning}

GKN is a standard for ergonomic requirements for an operator's workload. The document complying with EuropeanCouncilDirective 90/269 / EEC also includes additional requests from the authorities for the working environment in AFS 2012: 2 on ergonomics for the prevention of musculoskeletal disorders. It also refers to the Swedish working environment authorities Organizatorisk och social arbetsmiljö. AFS 2015: 4 and Arbets platsens utformning. AFS 2009: 02.

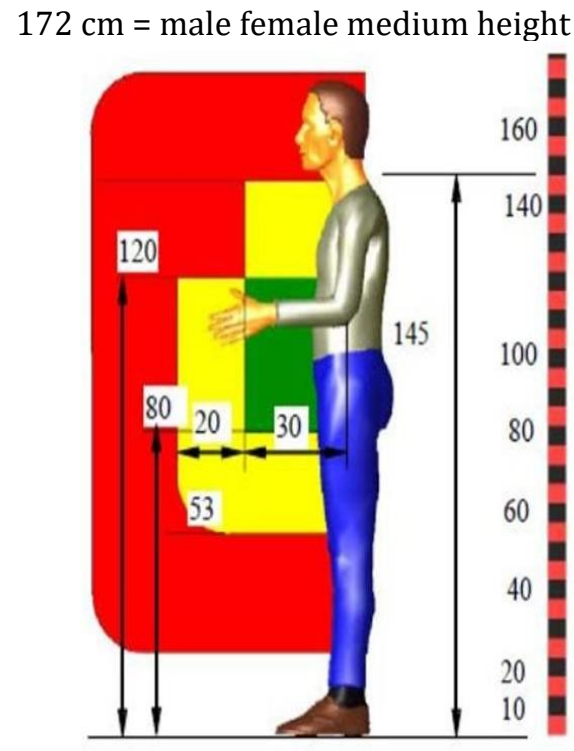

Figure 9

Standards include measurements of desirable jobs and those jobs that have been avoided. Figure 9 shows an example of three different areas. Green is the area where you work, and red is the least desired position, related to the position of the hands. There is a high risk of injury when the hands are in the red areas for more than 2 hours or more than 100 times a day.

The robot should have enough sensory subsystems to be able to handle the details in a good way. An industrial robot is usable because it is reprogrammable and can be integrated into a larger system connected to intelligent sensors and subsystems etc.

The robot's operating space must be divided into 3 zones in which the robot can move at different speeds and these zones will be controlled by sensors. 
The first area is where the robot touches the workpiece. In this area the robot must be able to move quickly and there should be no operator access to this area as long as the robot is in working mode.

The second area is where the robot moves the part into the human workspace. There should be sensors in this area to ensure that there is no human interference on the way when the robot is moving the object, otherwise the robot should stop or slow down.

The third area is the collaborative workspace in which both the human and the robot move. In this space the robot should move very slowly, and the movements must be controlled by man and also be able to be stopped quickly by touching the robot.

The collaborative robot (COBOT) must be able to move in the common workspace only if the movements are small. For larger movements, such as turning, the man should take a step and let the robot move first from the common workspace and move backwards before returning to the workspace. The sensors can also be placed on all operator instruments.

\section{Conclusions}

The trends in the manufacturing industry are moving towards the seamless integration of the world's physical and digital data to enable rapid integration, feedback and control loops throughout the distribution of production infrastructure. This article presents experimental models for intelligent COBOT type platform within the NUCLEUS project "Interdisciplinary research on the design and implementation of a multi-application intelligent robotic platform type COBOT of ultra-precise telemetry and remote control, for the Smart Industry (4.0)".

\section{References}

[1] Gheorghe I. Gh., "The Development of Intelligent Industry in Romania", International Journal of Modeling and Optimization, Vol. 10, No. 6, December 2020;

[2] Constantin A., „Telementenance and Teleservice Oriented Design of Dependable Mechatronic System in Automotive Industry", Proceedings of the International Conference on Numerical Analysis and Applied Mathematics 2014 (ICNAAM-2014), AIP Conference Proceedings 1648, 620004 (2015); doi: http://dx.doi.org/10.1063/1.4912854;
[3] Abrahamson, S., Ryder, P., Unterberg, B., Crowdstorm: The Future of Innovation, Ideas, and Problem Solving, John Wiley \& Sons, Inc. 2013;

[4] Gheorghe I. Gh., "Original Concepts and Achievements for Designing of Smart Mechatronics and Cyber-Mixmechatronics Systems used in Laboratories and in the Industry", IFAC PapersOnLine 51-30 (2018) 598-603

[5] Gheorghe I. Gh., "Cyber-Mixmechatronics Mix Concepts, Integrated IN Cobotic Platforms for the Smart Industry (4.0) and the Digitized Enterprise", International Journal of Mechatronics and Applied Mechanics, 2019, Issue 6, Vol. I, pp. 165-179

[6] Gheorghe I. Gh., „Vision of the National Institute of Research-Development for Mechatronics and Measurement Technique - INCDMTM Bucharest in research and innovation for the smart domain "Internet of Things -IoT", integrated in the COBOT technology platforms", International Journal of Modeling and Optimization (IJMO), IJMO 2019 Vol.9(5): 291-297 ISSN: 2010-3697 DOI: 10.7763/IJMO.2019.V9.725;

[7] Machado, J, Soares, F., Celina, L: A virtaul Workbench applied to Automation Students Response Analysis Proceeings of Controlo 2014, Portugal, Vol: 321, Pages 709-719, Published: 2015; DOI:10.1007/978-3-319-10380-8_68;

[8] Rizescu, C.I., Udrea, C. Rizescu, D., Experimental Setup for Harmonic Drive Efficiency Determination, Advanced Materials Research Vols. 463-464 (2012) pp. 1518-1521, ISSN 10226680, ISBN 978-303785363-4;

[9] Varela, M.L.R., Putnik, G.D., Manupati, V.K., Rajyalakshmi, G., Trojanowska, J., Machado, J. (2018), "Collaborative manufacturing based on cloud, and on other I4.0 oriented principles and technologies: A systematic literature review and reflections", Management and Production Engineering Review Vol. 9, Nr. 3, Sept. 2018 • pp. 90-99, DOI: 10.24425/119538;

[10] Voicu A., Gheorghe I. Gh., „Complex 3D Measuring by multiple laser scaning of automotive parts", Advanced Materials, Volume 837, 2013, pp. 511-516, DOI: 10.4028/www.scientific.net/AMR.837.511 